\title{
HIGHER APPENDICULAR AND TRUNK FAT MASS USING BIOELECTRICAL IMPEDANCE ANALYSIS ARE RELATED TO HIGHER RESTING BLOOD PRESSURE IN OLDER ADULTS
}

\author{
D. Takagi ${ }^{1}$, M. Kageyama ${ }^{2}$, S. Kojima ${ }^{3}$, Y. Nishida ${ }^{4}$
}

\begin{abstract}
Background: Little is known about how fat mass and muscle mass in different parts of the body (e.g., appendages, trunk) using bioelectrical impedance analysis influences resting blood pressure in older adults. Objective: The purpose of the study was to clarify the association between resting blood pressure and muscle mass and fat mass in older adults using bioelectrical impedance analysis. Design: A cross-sectional study. Settings: A sample living independently in the community. Participants: The subjects were older adults between the ages of 65 and 85 years $(n=100)$. Measurements: Systolic, diastolic and mean arterial pressure was measured using an automatic hemodynamometer, and bioelectrical impedance analysis was used to estimate muscle mass and fat mass. Results: A positive correlation was observed between total fat mass, left and right arm fat mass, trunk fat mass, and left and right leg fat mass and resting systolic, diastolic and mean arterial pressure $(p<0.05)$, but this was not observed with any muscle mass $(p>0.05)$. In a multiple regression analysis adjusted for sex, systolic, diastolic and mean arterial pressure were independently predicted by total fat mass, left and right arm fat mass, trunk fat mass, and left and right leg fat mass ( $<<0.05)$. Conclusions: These findings suggest that total, appendicular, and trunk fat mass, measured using bioelectrical impedance analysis, could aid in detecting the factors that increase blood pressure in clinical settings and even in daily life, thereby helping in controlling blood pressure.
\end{abstract}

Key words: Blood pressure, muscle mass, fat mass, bioelectrical impedance analysis, older adults.

\section{Introduction}

Ischaemic heart disease and stroke were the major leading causes of death in the world in 2012 (1). Age, particularly being over the age of 65 years, is one of the main risk factor for stroke and heart disease (2-3). $51 \%$ of deaths from stroke (cerebrovascular disease) and $45 \%$ of deaths from ischemic heart disease are attributable to high blood pressure (4). Hypertension leads to the development of arterial stiffness, and cardiovascular diseases, accordingly (5). The prevalence of hypertension in older adults aged 60 years or over is $67 \%$ (6), and the residual lifetime risk for hypertension in middle-aged and older adults is $90 \%$ (7). That is, the prevalence of hypertension in older adults is particularly high. If the prevention of hypertension which is related to developing about half the number of stroke (cerebrovascular disease) and ischemic heart disease (4)

1. Department of Physical Therapy, Health Science University; 2. Department of Rehabilitation, Toyoda Eisei Hospital; 3. Department of Rehabilitation, Suzukake Healthcare Hospital; 4. Department of Physical Therapy, Seirei Christopher University.

Corresponding Author: Daisuke Takagi, Department of Physical Therapy, Health Science University: 7187 Kodachi, Fujikawaguchiko-Town, Yamanashi, 401-0380, Japan. TEL : +81 555-83-5299/FAX : +81 555-83-5298, Email : pt.takadai@gmail.com in older adults is possible, it may potentially lead to a decrease in the number of cardiovascular related deaths.

Many studies have investigated the risk factors associated with hypertension. For example, Han et al reported that subjects aged 60 years or older with sarcopenic obesity had a greater risk of hypertension; sarcopenic obesity was defined as an appendicular muscle mass/weight $<1$ standard deviation (SD) below the mean of a sample of healthy adults (aged 20-40 years) and a body mass index (BMI) of $\geq 25 \mathrm{~kg} / \mathrm{m} 2$ (8). Park et al also suggested that sarcopenic obesity is associated with hypertension (9). Therefore, higher fat mass and lower muscle mass may lead to higher resting blood pressure. Moreover, another study found that central but not peripheral fat mass percentage was associated with high blood pressure in older adults (10). Pulse wave velocity has also been found to be an independent predictor of incident hypertension (11) and is related to appendicular muscle mass decline (12).

Fat mass and muscle mass can be measured using dual-energy x-ray absorption (DEXA) and CT scan. Measurement of fat mass and muscle mass using DEXA or CT scan is inconvenient in clinical settings or daily life. The bioelectrical impedance analysis (BIA) device is widely accepted as a safe, rapid, low cost, highly reliable, 
and valid technique to estimate muscle mass and fat mass (13-16), and the relationship between body lean mass, body fat, and visceral fat areas measured by DEXA and CT scans (17-19). However, little is known about how fat mass and muscle mass in different parts of the body (e.g., appendages, trunk) using BIA influences resting blood pressure in older adults. The measurement of BIA is more convenient compared with those of DEXA and CT scans, and clinicians and subjects can purchase BIA devices inexpensively for use in clinical settings or daily life. Clarifying the relationship between fat mass and muscle mass in the appendages and trunk using BIA and resting blood pressure could aid in detecting the factors that increase blood pressure in clinical settings and even in daily life, which will help control blood pressure, thereby preventing cardiovascular events.

The purpose of this study was to clarify the association between resting systolic blood pressure, diastolic blood pressure, mean arterial pressure, total muscle mass, total fat mass, appendicular muscle mass and fat mass, trunk muscle mass and fat mass in older adults in the community using BIA. We hypothesized that fat mass and muscle mass would influence on resting blood pressure.

\section{Methods}

\section{Subjects}

In this cross-sectional study, subjects were older adults ( $\mathrm{n}=100$, male: 47 , female: 53 ) visiting an outpatient internal medicine clinic and living independently in the community; their age was $65-85$ years $(74.9 \pm 5.3)$. Subjects were excluded if 1) they had a pacemaker and/ or 2) they had a systolic blood pressure of greater than $180 \mathrm{mmHg}$ and/or diastolic blood pressure of greater than $100 \mathrm{mmHg}$ (20). According to the medical records, 91 subjects had hypertension and had been using antihypertensive drugs, including Calcium antagonists, angiotensin II receptor antagonists, angiotensin converting enzyme inhibitors, $\alpha$ blockers, $\beta$ blockers, $\alpha \beta$ blockers, diuretics, renin inhibitors, angiotensin II receptor blocker/calcium channel blocker combination medication, angiotensin II receptor blocker/diuretics combination medication, and amlodipine besilate/ atorvastatin calcium hydrate combination medication (Table 1). All subjects read and signed an informed consent form and this study was approved with the Ethics Committee of Seirei Christopher University.

\section{Blood Pressure}

Systolic and diastolic pressures were measured using an automatic hemodynamometer (HEM-907, Omron Health Care, Kyoto, Japan) after subjects sat for a 5-min rest period. Blood pressure was measured twice, and the average of the values was recorded as the systolic and diastolic pressures. Mean arterial pressure [(systolic blood pressure - diastolic blood pressure)/3 + diastolic blood pressure] were calculated. The measurements were recorded from the left arm at the height of the heart.

Table 1

Characteristics of the study subjects $(n=100)$

\begin{tabular}{|c|c|}
\hline Characteristics & Mean (SD) \\
\hline Age (years) & $74.9(5.3)$ \\
\hline Height $(\mathrm{cm})$ & $152.2(9.1)$ \\
\hline Weight (kg) & $52.4(9.9)$ \\
\hline BMI (kg/m2) & $22.6(2.8)$ \\
\hline SBP (mmHg) & $134.1(17.3)$ \\
\hline $\mathrm{DBP}(\mathrm{mmHg})$ & $73.8(9.9)$ \\
\hline MAP (mmHg) & $93.9(11.0)$ \\
\hline PP (mmHg) & $60.3(14.2)$ \\
\hline Total muscle mass (kg) & $35.8(8.0)$ \\
\hline Left arm muscle mass (kg) & $2.3(0.6)$ \\
\hline Right arm muscle mass (kg) & $2.3(0.6)$ \\
\hline Trunk muscle mass (kg) & $18.0(3.7)$ \\
\hline Left leg muscle mass (kg) & $6.7(1.5)$ \\
\hline Right leg muscle mass (kg) & $6.6(1.5)$ \\
\hline Total fat mass (kg) & $13.5(4.0)$ \\
\hline Left arm fat mass $(\mathrm{kg})$ & $0.9(0.3)$ \\
\hline Right arm fat mass (kg) & $0.9(0.3)$ \\
\hline Trunk fat mass $(\mathrm{kg})$ & $6.9(2.1)$ \\
\hline Left leg fat mass (kg) & $2.4(0.7)$ \\
\hline Right leg fat mass (kg) & $2.4(0.7)$ \\
\hline \multicolumn{2}{|l|}{ Use of Antihypertensive drugs } \\
\hline Ca antagonists $(\%)$ & 49 \\
\hline Angiotensin II receptor antagonists (\%) & 61 \\
\hline Angiotensin converting enzyme inhibitors (\%) & 8 \\
\hline$\alpha$ or $/$ and $\beta$ blockers $(\%)$ & 24 \\
\hline Diuretics $(\%)$ & 17 \\
\hline Renin inhibitor $(\%)$ & 3 \\
\hline Combination medication $(\%)$ & 20 \\
\hline
\end{tabular}

Values are expressed as means (SD) unless otherwise specified; BMI body mass index, SBP systolic blood pressure, DBP diastolic blood pressure; MAP mean arterial pressure, PP pulse pressure

\section{Muscle Mass and Fat Mass}

BIA with an ioi 3535 (Kobe Medi-care Co. Ltd) was used to estimate the total muscle mass, total fat mass, appendicular muscle mass and fat mass, and trunk muscle mass and fat mass. BIA is reliable and valid technique to estimate muscle mass and fat mass (15-16). 
Table 2

Correlation coefficient of blood pressure with muscle and fat mass $(\mathrm{n}=100)$

\begin{tabular}{lcccccc}
\hline Characteristics & \multicolumn{2}{c}{ SBP } & \multicolumn{2}{c}{ DBP } & \multicolumn{2}{c}{ MAP } \\
& $\mathbf{r}$ & $\mathbf{p}$ & $\mathbf{r}$ & $\mathbf{p}$ & $\mathbf{r}$ & $\mathbf{p}$ \\
\hline Total muscle mass & -0.04 & 0.679 & 0.09 & 0.391 & 0.03 & 0.766 \\
Left arm muscle mass & -0.06 & 0.556 & 0.03 & 0.765 & -0.01 & 0.897 \\
Right arm muscle mass & -0.07 & 0.521 & 0.02 & 0.847 & -0.02 & 0.825 \\
Trunk muscle mass & -0.02 & 0.830 & 0.12 & 0.236 & 0.06 & 0.550 \\
Left leg muscle mass & -0.03 & 0.801 & 0.08 & 0.441 & 0.03 & 0.741 \\
Right leg muscle mass & -0.04 & 0.661 & 0.06 & 0.546 & 0.01 & 0.895 \\
Total fat mass & 0.23 & 0.019 & 0.27 & 0.006 & 0.29 & 0.004 \\
Left arm fat mass & 0.25 & 0.012 & 0.31 & 0.002 & 0.32 & 0.001 \\
Right arm fat mass & 0.26 & 0.010 & 0.33 & $<0.001$ & 0.33 & $<0.001$ \\
Trunk fat mass & 0.23 & 0.023 & 0.27 & 0.006 & 0.28 & 0.004 \\
Left leg fat mass & 0.23 & 0.023 & 0.26 & 0.009 & 0.28 & 0.005 \\
Right leg fat mass & 0.23 & 0.020 & 0.27 & 0.007 & 0.28 & 0.004 \\
\hline
\end{tabular}

SBP systolic blood pressure, DBP diastolic blood pressure, MAP mean arterial pressure

Impedance values for seven segments (total muscle and fat mass, appendicular muscle and fat mass, trunk muscle and fat mass) were measured at $5 \mathrm{~Hz}, 50 \mathrm{~Hz}$, and $250 \mathrm{~Hz}$ by the tetra-polar method using 8 touch electrodes. In the tetra-polar method, the current electrode (to send the electric current) and voltage electrode (to measure the impedance of human body) are separated and currents through each electrode are measured, thereby reducing contact resistance. Current and voltage electrodes are situated at both handle sensors and foot sensors of this device (eight electrodes in all). For the analysis, subjects stood upright barefoot on the device. Their body weight was automatically measured, and then we entered their name, age, sex, and height into the analyzer. Subjects grasped the handles, and their palms and soles of their feet were in contact with the current and voltage electrodes.

\section{Statistical Analysis}

A priori power analysis for correlation and linear multiple regression using $G^{*}$ power 3.1.9.2 (Correlation; Two tails, Correlation @ H1 $=0.3, \alpha=0.05$, Power $=0.8$ : Linear multiple regression; Effect size $=0.15, \alpha=0.05$, Power $=0.8$, three variables ) determined minimal sample size to be 82 and 77 subjects, respectively. Statistical evaluation was performed using JMP 11 software (SAS Institute Japan, Tokyo, Japan). The results were expressed as the mean $\pm \mathrm{SD}$ and the significance was set at $\mathrm{p}<0.05$. Pearson correlations were used to evaluate the relationship between total muscle mass, total fat mass, appendicular muscle mass and fat mass, trunk muscle mass and fat mass and systolic blood pressure, diastolic blood pressure and mean arterial pressure. Moreover, associations between total muscle mass, total fat mass, appendicular muscle mass and fat mass, trunk muscle mass and fat mass with systolic blood pressure, diastolic blood pressure and mean arterial pressure were evaluated in a multiple regression analysis adjusted for the following multiple confounders: age and/ or sex.

\section{Results}

The characteristics of the study subjects are presented in Table 1. In the Pearson correlations, no correlation was observed between total muscle mass, left and right arm muscle mass, trunk muscle mass, left and right leg muscle mass and resting systolic blood pressure $(p>0.05$; see Table 2$)$, resting diastolic blood pressure $(p>0.05$; see Table 2) and resting mean arterial pressure ( $p>0.05$; see Table 2). However, a positive correlation was observed between total fat mass, left and right arm fat mass, trunk fat mass, left and right leg fat mass and resting systolic blood pressure $(p<0.05$; see Table 2$)$, resting diastolic blood pressure $(\mathrm{p}<0.05$; see Table 2$)$ and resting mean arterial pressure $(p<0.05$; see Table 2$)$. Age was not significantly associated with total fat mass, left and right arm fat mass, trunk fat mass, left and right leg fat mass $(\mathrm{r}=-0.07, \mathrm{r}=-0.07, \mathrm{r}=-0.06, \mathrm{r}=-0.10, \mathrm{r}=-0.08, \mathrm{r}=-0.07$, $\mathrm{p}>0.05)$. In the multiple regression analysis adjusted for sex, systolic, diastolic and mean arterial pressure was independently predicted by total fat mass, left and right arm fat mass, trunk fat mass, left and right leg fat mass ( $p$ $<0.05$; see Table 3). 
Table 3

Multiple regression analysis of total fat mass, appendicular fat mass, and systolic, diastolic and mean arterial blood pressure after adjusting for sex $(n=100)$

\begin{tabular}{|c|c|c|c|c|c|c|}
\hline Characteristics & \multicolumn{2}{|c|}{ SBP } & \multicolumn{2}{|c|}{ DBP } & \multicolumn{2}{|c|}{ MAP } \\
\hline Total fat mass $(\mathrm{kg})$ & 0.22 & 0.026 & 0.28 & 0.006 & 0.28 & 0.005 \\
\hline Left arm fat mass (kg) & 0.24 & 0.016 & 0.31 & 0.002 & 0.32 & 0.002 \\
\hline Right arm fat mass (kg) & 0.25 & 0.014 & 0.33 & $<0.001$ & 0.33 & $<0.001$ \\
\hline Trunk fat mass (kg) & 0.22 & 0.033 & 0.28 & 0.006 & 0.28 & 0.005 \\
\hline Right leg fat mass (kg) & 0.22 & 0.027 & 0.27 & 0.007 & 0.28 & 0.005 \\
\hline
\end{tabular}

SBP systolic blood pressure, DBP diastolic blood pressure, MAP mean arterial pressure

\section{Discussion}

Although no correlation was observed between total muscle mass, appendicular muscle mass, trunk muscle mass and resting systolic, diastolic and mean arterial pressure, total fat mass, appendicular fat mass and trunk fat mass correlated positively with systolic, diastolic and mean arterial pressure when measured using BIA. Thus, we suggest that higher total fat mass as well as appendicular and trunk fat mass can be related to higher systolic, diastolic and mean arterial pressure in older adults in the community. These findings suggest that total, appendicular, and trunk fat mass, measured using BIA, could be useful to identify the factors that increase blood pressure, thereby helping in controlling blood pressure and preventing cardiovascular events.

With respect to the association between muscle mass and blood pressure, we did not consider whether the subjects had sarcopenia or not. Han et al defined sarcopenic obesity as an appendicular muscle mass / weight $<1$ standard deviation (SD) below the mean of a sample of healthy adults (aged 20-40 years) and a body mass index (BMI) of $\geq 25 \mathrm{~kg} / \mathrm{m} 2$ (8). Hence specific muscle mass decreases may be necessary to influence blood pressure. In contrast, non-sarcopenic subjects (defined as decreasing fast twitch fibers) are more likely to have hypertension than sarcopenic subjects (21). Sadamoto et al reported that subjects with a higher ratio of fast twitch fibers had a higher blood pressure (22), and a lower ratio of slow twitch fibers raised resting blood pressure (23). The relationship between decreased fast twitch fibers and higher blood pressure may be complicated by other factors, but fat mass may be more modifiable than muscle mass with respect to blood pressure effects. Future studies may shed further light on these relationships as BIA measures the muscle mass of both fast and slow twitch fibers.

In this study, weak correlation was observed between fat mass and blood pressure. Blood pressure is determined by cardiac output by peripheral vascular resistance that may be confounded by multiple factors such as stroke volume, heart rate, vascular bed area, arterial wall elasticity, and blood viscosity etc. Therefore, the relationship between fat mass and blood pressure may be weak. BIA measurements are affected by meal or beverage consumed and exercise performed prior to the analysis (24-25). It has been also reported that consumption of food or fluid and exercise do not influence the measurement of body composition using BIA (26-27), but may cause fat mass to be under- or overestimated. Thus, lack of control for food or beverage consumption or exercise performed prior to BIA in our study may have led to the weak correlation between fat mass and blood pressure. Future studies are needed to further explore this phenomenon.

This study has some limitations. First, the sample size was small and the participants came from a small region. We measured fat mass, muscle mass, and blood pressure only among older Japanese adults, and our results may not be generalizable to people of other races and ages. Second, the cross-sectional design used in this study does not allow us to determine a causal relationship between fat mass and blood pressure. Therefore, the manner in which fat mass influences blood pressure remains unclear. Third, we did not modulate meal intake, hydration or exercise for subjects prior to BIA measurements. Therefore, we may have underestimated or overestimated the relationship between fat mass and blood pressure.

Funding: The sponsors had no role in the design and conduct of the study; in the collection, analysis, and interpretation of data; in the preparation of the manuscript; or in the review or approval of the manuscript.

\section{Conflict of Interest: None to declare}

Ethics Statement: All subjects read and signed an informed consent form and this study was approved with the Ethics Committee of Seirei Christopher University.

\section{References}

1. The World Health Organization. The top 10 causes of death. 2014. http:// www.who.int/mediacentre/ factssheets/fs310/en/. Aceessed 7 Apr 2015

2. Kelly-Hayes M. Influence of age and health behaviors on stroke risk: lessons from longitudinal studies. J Am Geriatr Soc 2010; 58: S325-328. 
3. Gorina Y, Lentzner H. Multiple causes of death in old age. Aging Trends 2008; 9: 1-9.

4. The World Health Organization. GLOBAL HEALTH RISKS; Mortality and burden of disease attributable to selected major risks. 2009; 1-62.

5. Lee HY, Oh BH. Aging and arterial stiffness. Circ J 2010; 74: 2257-62.

6. Ostchega Y, Dillon CF, Hughes JP, Carroll M, Yoon S. Trends in hypertension prevalence, awareness, treatment, and control in older U.S. adults: data from the National Health and Nutrition Examination Survey 1988 to 2004. J Am Geriatr Soc 2007; 55: 1056-1065.

7. Vasan RS, Beiser A, Seshadri S, et al. Residual lifetime risk for developing hypertension in middle-aged women and men: The Framingham Heart Study. JAMA 2002; 287: 1003-1010.

8. Han K, Park YM, Kwon HS, et al. Sarcopenia as a determinant of blood pressure in older Koreans: Findings from the Korea National Health and Nutrition Examination Surveys (KNHANES) 2008-2010. PLoS One 2014; 9: 1-7.

9. Park SH, Park JH, Song PS, et al. Sarcopenic obesity as an independent risk factor of hypertension. J Am Soc Hypertens 2013; 7: 420-425.

10. van Dijk S, van den Meiracker A, van der Cammen T, Mattace Raso F, van der Velde N. Central but not peripheral fat mass percentage is associated with blood pressure components in the elderly. Age Ageing 2012; 41: 534-540.

11. Najjar SS, Scuteri A, Shetty V, et al. Pulse wave velocity is an independent predictor of the longitudinal increase in systolic blood pressure and of incident hypertension in the Baltimore Longitudinal Study of Aging. J Am Coll Cardiol 2008; 51: 1377-1383.

12. Abbatecola AM, Chiodini P, Gallo C, et al. Pulse wave velocity is associated with muscle mass decline: Health ABC study. Age (Dordr) 2012; 34: 469-478.

13. Janssen I, Heymsfield SB, Baumgartner RN, Ross R. Estimation of skeletal muscle mass by bioelectrical impedance analysis. J Appl Physiol (1985) 2000; 89: 465-471.

14. Pietiläinen KH, Kaye S, Karmi A, Suojanen L, Rissanen A, Virtanen KA. Agreement of bioelectrical impedance with dual-energy X-ray absorptiometry and MRI to estimate changes in body fat, skeletal muscle and visceral fat during a 12-month weight loss intervention. Br J Nutr 2013; 109: 1910-1916.

15. Fornetti WC, Pivarnik JM, Foley JM, Fiechtner JJ. Reliability and validity of body composition measures in female athletes. J Appl Physiol (1985) 1999; 87: 1114-22.
16. Miyatani M, Kanehisa $\mathrm{H}$, Masuo $\mathrm{Y}$, Ito M, Fukunaga T. Validity of estimating limb muscle volume by bioelectrical impedance. J Appl Physiol(1985) 2001; 91: 386-94.

17. Svendsen OL, Haarbo J, Heitmann BL, Gotfredsen A, Christiansen C. Measurement of body fat in elderly subjects by dual-energy x-ray absorptiometry, bioelectrical impedance, and anthropometry. J Clin Nutr 1991; 53: 1117-23.

18. Ling $\mathrm{CH}$, de Craen $\mathrm{AJ}$, Slagboom PE, et al. Accuracy of direct segmental multi-frequency bioimpedance analysis in the assessment of total body and segmental body composition in middle-aged adult population.Clin Nutr 2011; 30: 610-5.

19. Nagai M, Komiya H, Mori $\mathrm{Y}$, et al. Estimating visceral fat area by multifriequency bioelectrical impedance. Diabetes Care 2010; 33: 1077-9.

20. Guidelines for Rehabilitation in Patients with Cardiovascular Disease (JCS 2012), 1-61.

21. Landi F, Liperoti R, Russo A, et al. Sarcopenia as a risk factor for falls in elderly individuals: results from the ilSIRENTE study. Clin Nutr 2012; 31: 652-658.

22. Sadamoto T, Mutoh Y, Miyashita M. Cardiovascular reflexes during sustained handgrip exercise: role of muscle fiber composition, potassium and lactate. Eur J Appl Physiol Occup Physiol 1992; 65: 324-330.

23. Hernelahti M, Tikkanen HO, Karjalainen J, Kujala UM. Muscle fiber-type distribution as a predictor of blood pressure: a 19-year follow-up study. Hypertension 2005; 45: 1019-1023.

24. Kushner RF, Gudivaka R, Schoeller DA. Clinical characteristics influencing bioelectrical impedance analysis measurements. Am J Clin Nutr 1996; 64: 423427.

25. Gallagher M, Walker KZ, O'Dea K. The influence of a breakfast meal on the assessment of body composition using bioelectrical impedance. Eur J Clin Nutr 1998; 52: 94-7.

26. Vilaca KH, Ferriolli E, Lima NK, Paula FJ, Moriquti JC. Effect of fluid and food intake on the body composition evaluation of elderly persons. J Nutr Health Aging 2009; 13: 183-6.

27. Andreacci JL, Dixon CB, Laqomarsine M, Ledezma C, Goss FL, Robertson RJ. Effect of a maximal treadmill test on percent body fat using leg-to-leg bioelectrical impedance analysis in children. J Sports Med Phys Fitness 2006; 46: 454-7. 